\title{
Is There Scale-Dependent Bias in Single-Field Inflation?
}

\author{
Roland de Putter, ${ }^{\star}$ Olivier Doré, $\star$ and Daniel Green ${ }^{\star}$

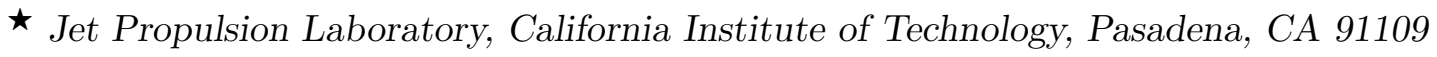 \\ California Institute of Technology, Pasadena, CA 91125 \\ * Canadian Institute for Theoretical Astrophysics, Toronto, ON M5S 3H8, Canada \\ Canadian Institute for Advanced Research, Toronto, ON M5G 1Z8, Canada
}

\begin{abstract}
Scale-dependent halo bias due to local primordial non-Gaussianity provides a strong test of singlefield inflation. While it is universally understood that single-field inflation predicts negligible scale-dependent bias compared to current observational uncertainties, there is still disagreement on the exact level of scale-dependent bias at a level that could strongly impact inferences made from future surveys. In this paper, we clarify this confusion and derive in various ways that there is exactly zero scale-dependent bias in single-field inflation. Much of the current confusion follows from the fact that single-field inflation does predict a mode coupling of matter perturbations at the level of $f_{\mathrm{NL}}^{\text {local }} \approx-5 / 3$, which naively would lead to scale-dependent bias. However, we show explicitly that this mode coupling cancels out when perturbations are evaluated at a fixed physical scale rather than fixed coordinate scale. Furthermore, we show how the absence of scale-dependent bias can be derived easily in any gauge. This result can then be incorporated into a complete description of the observed galaxy clustering, including the previously studied general relativistic terms, which are important at the same level as scale-dependent bias of order $f_{\mathrm{NL}}^{\text {local }} \sim 1$. This description will allow us to draw unbiased conclusions about inflation from future galaxy clustering data.
\end{abstract}




\section{Contents}

1 Introduction $\quad 2$

2 The Observed Galaxy Bias in Single-Field Inflation $\quad 4$

3 Single-Field Consistency and Derivations of Scale-Dependent Bias 7

$\begin{array}{lll}3.1 & \text { Single-field consistency conditions } & 7\end{array}$

3.2 Scale-dependent bias from mode coupling 10

3.3 Scale-dependent bias from direct application of consistency conditions 13

4 Summary and Discussion $\quad 14$

A Consistency Conditions in Newtonian and Synchronous Gauge 16

B Second Order Perturbation Theory Calculation of Mode Coupling 18

$\begin{array}{ll}\text { References } & 21\end{array}$ 


\section{Introduction}

Primordial non-Gaussianity is a powerful probe of the physics of inflation. In particular, local type non-Gaussianity can be used to discriminate between single-field and multi-field models of inflation. Single-field models generally predict negligible local non-Gaussianity $[1,2], f_{\mathrm{NL}}^{\text {local }} \sim$ $\left(n_{s}-1\right) \ll 1$, such that any detection of non-zero local non-Gaussianity would rule out the singlefield scenario. On the other hand, multi-field models quite generically predict $\left|f_{\mathrm{NL}}^{\text {local }}\right| \gtrsim 1[3-5]$, setting a natural target for the precision of primordial non-Gaussianity searches at $\sigma\left(f_{\mathrm{NL}}^{\text {local }}\right) \lesssim 1$.

Currently, the best constraint on local non-Gaussianity comes from the cosmic microwave background $(\mathrm{CMB})$ temperature and polarization bispectra, $f_{\mathrm{NL}}^{\text {local }}=0.8 \pm 5.0(68 \%$ confidence level) [6], thus showing no evidence for deviations from Gaussianity, while being about an order of magnitude away from the order unity target precision. Unfortunately, due to the limited number of modes accessible with the CMB, it will not be possible to improve this error bar by more than a factor of two with CMB data only [7]. Fortunately, local primordial non-Gaussianity also leads to a strong signature in cosmological large-scale structure, in the form of a scale-dependent halo bias $\Delta b_{\mathrm{NG}}(k) \propto k^{-2}[8]$. The signal-to-noise of this effect is thus peaked on very large scales and therefore is safely distinguishable from the non-linearities of structure formation. While systematics in the large-scale galaxy clustering measurement present a challenge, it has been shown that large-volume future galaxy surveys can in principle use this scale-dependent bias to reach the target precision $\sigma\left(f_{\mathrm{NL}}^{\text {local }}\right) \lesssim 1[9-14]$.

Since an order unity precision measurement of $f_{\mathrm{NL}}^{\text {local }}$ is thus feasible, testing single-field inflation will require an equally precise prediction for scale-dependent bias in that class of models. However, currently, there is still disagreement in the literature about whether the scale-dependent bias is identically zero, or instead non-zero at the level of an effective non-Gaussianity of order unity. The difference in these predictions is small compared to current observational error bars, both amounting to the statement that single-field inflation predicts negligible scale-dependent bias, but for future surveys it is essential to settle this question. For example, if the theoretical prediction is off by order unity, we might end up falsely interpreting a measurement consistent with single-field inflation as evidence for multi-field inflation.

The main focus of this paper is to resolve the confusion about scale-dependent bias in singlefield inflation and to derive a consistent answer in multiple ways. The main conclusion will be that there is exactly zero scale-dependent bias in these models so that any future detection of scale-dependent bias would indeed rule out single-field inflation.

The confusion in the literature derives from the contrast between two types of arguments. On the one hand, the single-field consistency conditions are equivalent to stating that, locally, a large-scale curvature fluctuation has no physical effect on small-scale physics and is simply equivalent to a coordinate transformation. This strongly suggests (correctly) that there can be no modulation of the halo number density by the long mode and therefore no scale-dependent bias [15-21]. On the other hand, matter perturbations in synchronous gauge, for example, do see

a mode coupling at the level of an equivalent $f_{\mathrm{NL}}^{\text {local }} \sim-5 / 3$ [22], so that the small-scale variance is modulated by a large-scale mode, thus suggesting that there is scale-dependent bias [23-28]. We will resolve this paradox by noting that the latter is a modulation of the variance of matter perturbations on a fixed coordinate scale, but that the local halo number density can only depend 
on the variance on a given physical scale. We will then explicitly show that when the variance on a fixed physical scale is considered, the modulation cancels out, leaving no physical effect, and therefore no scale-dependent bias.

Moreover, we will explain how both the original order unity mode coupling and the later cancellation can be easily understood directly in terms of the single-field consistency conditions. Specifically, making use to the work of [29-31], it is easy to implement the initial conditions from single-field inflation in any gauge. The long wavelength mode can be introduced by a change of coordinates and therefore it is easy to determine its effect on any local quantity knowing only how it transforms under a diffeomorphism ${ }^{1}$. This basic observation has been applied to a number of large scale structure observables by a number of authors (see e.g. [34-44]). We apply this logic to the small-scale variance of matter perturbations to elegantly derive the mode coupling, but we will show that it can even be applied to the halo number density itself to directly obtain the result that $\Delta b_{\mathrm{NG}}(k) \equiv 0$ without referring to the modulation of the variance of matter perturbations.

In addition to a precise prediction for scale-dependent bias, there are other subtleties in the modeling of galaxy clustering, which, if not properly taken into account, could lead to misinterpretation of the data. What we observe in a survey are galaxy overdensities based on positions derived from observed redshifts and observed angular positions, which is not the same as the galaxy overdensity in some arbitrary given gauge. The difference includes redshift-space distortions, but also more subtle relativistic effects that contribute at order unity at the Hubble scale and thus are comparable in magnitude to the scale-dependent bias effect from $f_{\mathrm{NL}}^{\text {local }} \sim 1$. In addition, one has to be careful that the very definition of bias is gauge independent. These issues have all been addressed previously [45-47]. In this paper, we build on these results, in combination with the single-field prediction $\Delta b_{\mathrm{NG}}(k) \equiv 0$, to present a complete prescription for modeling the galaxy clustering that can be used to test primordial non-Gaussianity in an unbiased fashion.

The paper is outlined as follows. In Section 2, we will provide a recipe for the observed galaxy overdensity in single-field inflation that includes the proper relativistic definition of galaxy bias, the (absence of) single-field scale-dependent bias, and relativistic projection effects. This section serves as a summary of our main result and places it in the context of a complete galaxy clustering description. In Section 3, we will derive the scale-dependent bias in single-field inflation from several perspectives. We will reproduce many results that have been derived in the literate but we will show how they can be made compatible, leading to a single, consistent answer. We will conclude in Section 4.

The paper includes two appendices. In Appendix A, we show how to apply the inflationary consistency conditions in both Newtonian and synchronous gauge. We explain the slight differences that appear from each perspective. In Appendix B, we show explicitly that the mode coupling determined by second order perturbation theory is precisely the same as the one determined by the consistency conditions. In this sense, the results of Section 3 can be derived by a brute force calculation.

Finally, for the remainder of this article, we will drop the "local" superscript on $f_{\mathrm{NL}}$, as we will exclusively discuss local type non-Gaussianity.

\footnotetext{
${ }^{1}$ The same results can be derived in the Newtonian limit by [32, 33] using Galilean invariance.
} 


\section{The Observed Galaxy Bias in Single-Field Inflation}

We wish to unambiguously express, in the context of single-field inflation, the observed galaxy density perturbations in terms of underlying (first-order) cosmological perturbations that can be computed using Boltzmann codes such as CAMB. This problem can be divided into three components. First of all, even if we are given the fluctuations in physical galaxy number density in some gauge, $\delta_{g}$, we need to transform this to the observed galaxy density fluctuations, $\Delta_{g}$, which is estimated based on observed redshifts $z$ and angular positions $\hat{\mathbf{n}}$. Secondly, to obtain $\delta_{g}$, we need a way to define galaxy bias that does not suffer from gauge ambiguities. Finally, we need to specify the scale-dependence (if any) of the thus defined galaxy bias parameter.

The main focus of this paper is to compute and clarify the third component, i.e., is there a scale-dependent galaxy bias in single-field inflation? We will discuss this in detail in Section 3. In the current section, we simply use the main result of Section 3 as part of a complete recipe for the observed galaxy clustering in the context of single-field inflation, containing all three of the components discussed above.

First, the relation between observed galaxy overdensity and the density and metric perturbations in a given gauge has been addressed definitively in the literature, e.g. [45-52]. For instance, in terms of synchronous gauge fluctuations, where (in a spatially flat universe)

$$
d s^{2}=a^{2}(\tau)\left[-d \tau^{2}+(1-2 \hat{\psi}) \delta_{i j}^{\mathrm{K}} d x^{i} d x^{j}+\partial_{i} \partial_{j} \chi d x^{i} d x^{j}\right],
$$

and where velocity perturbations in cold dark matter are set to zero, [46] gives (borrowing here some notation from [52])

$$
\Delta_{g}(\hat{\mathbf{n}}, z)=\Delta_{g, l o c}(\hat{\mathbf{n}}, z)+\Delta_{g, \kappa}(\hat{\mathbf{n}}, z)+\Delta_{g, I}(\hat{\mathbf{n}}, z),
$$

with the "local" contribution,

$$
\begin{aligned}
\Delta_{g, \text { loc }} & =\delta_{g}+\frac{1}{2}\left[b_{e}-(1+2 \mathcal{Q})+\frac{1+z}{H} \frac{d H}{d z}-\frac{2}{D}(1-\mathcal{Q}) \frac{1+z}{H}\right]\left(\partial_{\|} \chi^{\prime}+\chi^{\prime \prime}\right) \\
& -\frac{1+z}{2 H} \partial_{\|}^{2} \chi^{\prime}-\frac{2}{D}(1-\mathcal{Q})\left(D \hat{\psi}+\frac{1}{2} \chi^{\prime}\right)
\end{aligned}
$$

and $\Delta_{g, \kappa}$ and $\Delta_{g, I}$ given in [52] as line-of-sight integrals over metric perturbations. We ignore the stochastic noise component here. For this expression to be valid, it is crucial that the perturbations on the right hand side are evaluated in synchronous gauge (of course, $\Delta_{g}$ can in principle be expressed in terms of perturbations in any gauge). In Eq. (2.3), $\delta_{g}$ is the physical galaxy overdensity in synchronous gauge, $b_{e}$ the evolution bias, $b_{e}=d \ln \left(a^{3} \bar{n}_{g}\right) / d \ln a$ (with $\bar{n}_{g}$ the mean physical number density), $\mathcal{Q}$ is the response to magnification $\delta \mathcal{M}$, defined such that magnification bias causes $\Delta_{g} \rightarrow \Delta_{g}+\mathcal{Q} \delta \mathcal{M}$, and $D$ is comoving distance to the galaxy sample.

Second, we need to relate the galaxy overdensity $\delta_{g}$ to the underlying matter (and metric) perturbations by a bias prescription. The standard approach is to define the galaxy bias $b$ as the proportionality factor between $\delta_{g}$ and the matter overdensity $\delta$. However, this makes the definition of $b$ gauge-dependent. The natural gauge choice is the synchronous gauge (e.g. [46]), 
as here slices of constant coordinate time $\tau$ corresponds to constant proper time so that there is no evolution bias. Thus,

$$
\delta_{g}=b \delta \quad \text { (synchronous gauge) }
$$

defines the bias. In an arbitrary gauge, physical density contrast is replaced by a "gauge invariant" quantity

$$
\delta_{g}^{\text {Invariant }} \equiv \delta_{g}+\frac{d \ln \bar{n}_{g}}{d \ln a} a H \delta \tau=b(\delta-3 a H \delta \tau),
$$

where $\delta \tau$ is the coordinate time shift between synchronous gauge and the gauge under consideration $^{2}$. Note that the ratio $\delta_{g} / \delta$ is clearly gauge-dependent. In particular, if the true bias $b$ as defined above is scale-independent, the ratio $\delta_{g} / \delta$ in a gauge where constant coordinate time slices do not correspond to constant proper time will in general be scale-dependent. It is thus important that we have first defined $b$ in a gauge-independent manner before we address scale-dependent bias.

The third component that needs to be addressed then is the specification of the galaxy bias $b$ defined in Eq. (2.4), and specifically its scale-dependence. To linear order, and for Gaussian initial conditions, the bias is scale-independent and can thus be described by a single free parameter, $b=b_{1}$. However, in the presence of primordial non-Gaussianity, there is an additional, scaledependent bias contribution. Specifically, if the non-Gaussianity is of local type, a large-scale potential fluctuation will modulate the initial variance of matter fluctuations on small scales, thus modulating the number density of halos that these fluctuations will collapse into at a later time. Working in synchronous gauge, and going to Fourier space for convenience,

$$
\delta_{g, l}=b_{1} \delta_{l}+\frac{d \ln n_{g}}{d \ln \sigma_{R}} \frac{d \ln \sigma_{R}}{d \varphi_{l}} \varphi_{l}=\left(b_{1}-\frac{3 H_{0}^{2} \Omega_{m}}{2 T(k) D(z) k^{2}} \frac{d \ln n_{g}}{d \ln \sigma_{R}} \frac{d \ln \sigma_{R}}{d \varphi_{l}}\right) \delta_{l} .
$$

Here, $\varphi$ is the metric perturbation evaluated in the early universe ${ }^{3}$, related to the matter overdensity $\delta$ at redshift $z$ by $k^{2} \varphi=-3 H_{0}^{2} \Omega_{m} / 2 T(k) D(z) k^{2}$ (using the Poisson equation), where $T(k)$ is the transfer function of matter perturbations, normalized to 1 at low $k, D(z)$ is the linear growth function, normalized such that $D(z)=1 /(1+z)$ during matter domination, and $\sigma_{R}^{2}$ is the initial variance of small-scale fluctuations smoothed over a comoving length $R$ enclosing the mass of the halos of interest (see Eq. 3.14). The quantity $n_{g}$ is the galaxy number density in a region with initial small-scale variance $\sigma_{R}^{2}$. Technically, we have thus assumed here that galaxies occupy halos of a single mass. More generally, the dependence on the variance $\sigma_{R}^{2}$ would be replaced by a dependence on the variance on a range of scales corresponding to a range of halo masses. We have applied an explicit subscript $l$ to stress that we are looking at the effect of large-scale fluctuations compared to the fluctuations that collapse into halos.

Thus, in the presence of mode coupling (non-zero $d \ln \sigma_{R} / d \varphi_{l}$ ), the bias gets a scale-dependent correction,

$$
b(k)=b_{1}+\Delta b_{\mathrm{NG}}(k),
$$

\footnotetext{
${ }^{2}$ i.e. $\tilde{\tau}=\tau+\delta \tau$, with $\tau$ the conformal time coordinate in synchronous gauge and $\tilde{\tau}$ the conformal time in the gauge under consideration

${ }^{3}$ The description here in terms of $\varphi_{l}$ is technically only correct if the initial conditions are taken during matter domination with the long mode $\varphi_{l}$ outside of the horizon, which is not possible of the mode entered the horizon during radiation domination. In Section 3, we will use a more general description in terms of the curvature perturbation $\zeta_{l}$, but here we use $\varphi_{l}$ since this is a very common description in the literature.
} 
where $\Delta b_{\mathrm{NG}}(k) \propto k^{-2}$. If we write the mode coupling in terms of an effective local non-Gaussianity parameter,

$$
\frac{d \ln \sigma_{R}}{d \varphi_{l}}=-2 f_{\mathrm{NL}}^{(\Delta b)},
$$

we obtain for the scale-dependent bias ${ }^{4}$,

$$
\Delta b_{\mathrm{NG}}(k)=2 f_{\mathrm{NL}}^{(\Delta b)} \frac{d \ln n_{g}}{d \ln \sigma_{R}} \frac{3 H_{0}^{2} \Omega_{m}}{2 T(k) D(z) k^{2}} .
$$

There has been significant confusion about the level of scale-dependent bias, i.e. of the mode coupling characterized by $f_{\mathrm{NL}}^{(\Delta b)}$ in Eq. (2.10), in single-field inflation. On the one hand, naively, $f_{\mathrm{NL}}^{(\Delta b)}$ is simply equal to the local non-Gaussianity parameter of $\zeta$ (the comoving curvature perturbation on slices of constant density), which in turn is small and given by the single-field consistency condition $[1,2]$, suggesting that the scale-dependent bias is

$$
f_{\mathrm{NL}}^{(\Delta b)} \stackrel{?}{=}-\frac{5}{12}\left(n_{s}-1\right),
$$

where $n_{s}$ is the tilt of the primordial power spectrum. On further inspection, since $\zeta$ and $\delta$ are non-linearly related, their non-Gaussianity parameters are not the same. In synchronous gauge, it has been found that the non-Gaussianity of matter perturbations is described by an effective non-Gaussianity parameter that gets shifted by $-5 / 3$ (e.g. [22]). This has led several authors to conclude that single-field inflation predicts scale-dependent bias of order unity [23-26] (see also $[27,28])$,

$$
f_{\mathrm{NL}}^{(\Delta b)} \stackrel{?}{=}-\frac{5}{3}-\frac{5}{12}\left(n_{s}-1\right) .
$$

On the other hand, however, basic physical arguments suggest that, modulo gradients, the metric perturbation $\varphi_{l}$ cannot have any effect on small-scale physics and its effect is equivalent to a coordinate transformation. This strongly suggests that the scale-dependent bias has to be zero [15-21]:

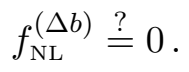

How are these result compatible? The main goal of this paper is to clarify this confusion. In the next section, we will calculate $\Delta b_{\mathrm{NG}}$ both using the mode coupling of density fluctuations in synchronous gauge and using the argument that $\varphi_{l}$ corresponds to a simple coordinate transformation, and will show that both approaches lead to the same result and that the former approach can be understood easily in terms of the latter. We aim to present a pedagogical presentation in order to settle the confusion as much as possible. The goal of the current section is simply to present the end result, which is that in single-field inflation, there is no scale-dependent bias:

$$
f_{\mathrm{NL}}^{(\Delta b)} \equiv 0
$$

\footnotetext{
${ }^{4}$ Note that if we assume a universal halo mass function, the response function of the galaxy number density simply becomes,

$$
\frac{d \ln n_{g}}{d \ln \sigma_{R}}=\left(b_{1}-1\right) \delta_{c}
$$

so that Eq. (2.10) reduces to the scale-dependent bias first discovered in [8]. 
In general, the observed galaxy clustering is thus fully described by Eqs (2.2) and Eq. (2.3), with the galaxy overdensity given by Eq. (2.4), and the bias by Eq. (2.7). In other words, in synchronous gauge, the galaxy overdensity entering Eq. (2.3) is $\delta_{g}(\mathbf{k})=\left(b_{1}+\Delta b_{\mathrm{NG}}(k)\right) \delta(\mathbf{k})$ so that in single-field inflation,

$$
\Delta b_{\mathrm{NG}}(k) \equiv 0 .
$$

Note however that the conversion from physical galaxy overdensity to observed galaxy overdensity in Eq. (2.3) does introduce terms that have the same scale-dependence as the scale-dependent bias would have. However, if after taking these well understood terms into account, there is remaining evidence for a true scale-dependent bias contribution $\Delta b_{\mathrm{NG}} \propto k^{-2}$, this would rule out single-field inflation.

While we have in this section expressed the observed galaxy overdensity in terms of synchronous gauge variables, our definition of bias was gauge invariant (Eq. (2.5)) so that our result can be applied to obtain $\Delta_{g}$ starting from any gauge. Existing calculations in Newtonian gauge can be written in terms of the same bias definition (see e.g. [47]) and therefore no special care is needed and we may again use $\Delta b_{\mathrm{NG}}=0$.

\section{Single-Field Consistency and Derivations of Scale-Dependent Bias}

In this section, we will provide several derivations of the result $\Delta b_{\mathrm{NG}}=0$ for initial conditions satisfying the single-field consistency relation. We will start by reviewing the inflationary singlefield consistency condition itself in Section 3.1. We will then derive the matter density mode coupling between long and short wavelengths using the same arguments that led to the inflationary consistency condition in Section 3.2 and we confirm the result by explicitly matching the second order perturbation theory solution of matter perturbations to the inflationary consistency relation (Appendix B). While we consistently find the same non-zero mode coupling in synchronous gauge, for example, it does not lead to scale-dependent bias because the modulation of short modes on a given physical scale is zero. We will show explicitly that the conversion from short modes on a fixed coordinate scale to modes on a fixed physical scale exactly cancels the aforementioned mode coupling and our derivation will make it clear that this cancellation is inevitable, as it is a direct manifestation of the consistency condition. We will finally see in Section 3.3 how this result can be derived more simply by applying the consistency conditions to the galaxies directly. All together, we will provide three different perspectives that can be applied in any gauge to derive the same physical result.

\subsection{Single-field consistency conditions}

In order to build intuition, let us first review the derivation of the consistency conditions for $\zeta$, the comoving curvature perturbation on slices of constant energy density [53, 54]. If we work on slices of constant density, on super-horizon scales (i.e. neglecting gradients of the curvature perturbation), the metric takes the general form,

$$
d s^{2}=a^{2}(\tau)\left[-d \tau^{2}+e^{2 \zeta(\mathbf{x})} d \mathbf{x}^{2}\right] .
$$

Dividing the curvature perturbation into a large- and small-scale contribution, $\zeta \equiv \zeta_{s}+\zeta_{l}$, the single-field consistency conditions are derived by observing that a large-scale perturbation can, 
to zeroth order in gradients of $\zeta_{l}$, be generated by a diffeomorphism. Specifically, we can locally describe the perturbations in a coordinate system (denoted by a tilde) where there is no large-scale mode,

$$
\tilde{d s}^{2}=a^{2}(\tau) e^{2 \tilde{\zeta}_{s}(\tilde{\mathbf{x}})} d \tilde{\mathbf{x}}^{2}
$$

where $\tilde{\zeta}_{s}(\tilde{\mathbf{x}})$, and more generally the local physics, knows nothing of the existence of a largescale mode. We have written only the space-space contribution to the line element (as there are only spatial metric perturbations in this gauge), and we do not apply a tilde to $\tau$ because we will not apply any transformation to it. The large-scale mode is then generated by applying a diffeomorphism to a new coordinate system (without a tilde), defined by the dilation,

$$
\mathbf{x} \equiv \tilde{\mathbf{x}}\left(1-\zeta_{l}\right)
$$

leaving the time coordinate unchanged. When $\zeta_{l}$ is a constant, this is a pure gauge mode. However, to leading order in derivatives we may modify $\zeta_{l} \rightarrow \zeta_{l}(\boldsymbol{x})$ and this matches onto a physical long wavelength solution up to higher derivative corrections. Since the line element itself is a scalar, in the new coordinate system, we then have, to first order in $\zeta_{l}$,

$$
d s^{2}=\tilde{d s^{2}}=a^{2}(\tau) e^{2 \tilde{\zeta}_{s}(\tilde{\mathbf{x}})} d \tilde{\mathbf{x}}^{2}=a^{2}(\tau) e^{2 \tilde{\zeta}_{s}(\tilde{\mathbf{x}})}\left(1+2 \zeta_{l}\right) d \mathbf{x}^{2}=a^{2}(\tau) e^{2\left(\tilde{\zeta}_{s}(\tilde{\mathbf{x}})+\zeta_{l}\right)} d \mathbf{x}^{2}
$$

where the final equality is true to first $\operatorname{order}^{5}$ in $\zeta_{l}$.

Comparing the far left-hand side of Eq. (3.4) to the far right hand-side shows first of all that the coordinate transformation indeed generates the large-scale curvature perturbation $\zeta_{l}$, and, secondly, that the small-scale curvature perturbation transforms as a scalar, i.e. $\zeta_{s}(\mathbf{x})=\tilde{\zeta}_{s}(\tilde{\mathbf{x}})$. Hence, in this $\mathbf{x}$ coordinate system where the large-scale mode is manifest,

$$
\zeta_{s}(\mathbf{x})=\tilde{\zeta}_{s}\left(\mathbf{x}\left(1+\zeta_{l}\right)\right)=\tilde{\zeta}_{s}(\mathbf{x})+\zeta_{l} \mathbf{x} \cdot \nabla \tilde{\zeta}_{s}(\mathbf{x})
$$

with $\tilde{\zeta}_{s}(\mathbf{x})$ statistically independent of $\zeta_{l}$. This is the squeezed limit, single-field consistency relation. Note that, in formulating the mode coupling, it was crucial that the short mode on the right-hand side is statistically independent of the long mode. This is equivalent to it being the small-scale component of a Gaussian field since for Gaussian fields, different scales are independent. Thus we could also write $\tilde{\zeta}_{s} \equiv \zeta_{s}^{G}$.

We now wish to relate the mode coupling in Eq. (3.5) to a non-Gaussianity parameter $f_{\mathrm{NL}}$. The usual ansatz is

$$
\zeta=\zeta^{G}+\frac{3}{5} f_{\mathrm{NL}}\left(\left(\zeta^{G}\right)^{2}-\left\langle\left(\zeta^{G}\right)^{2}\right\rangle\right)
$$

where $\zeta^{G}$ is a Gaussian field. This leads to the mode coupling,

$$
\zeta_{s}=\left(1+\frac{6}{5} \zeta_{l} f_{\mathrm{NL}}\right) \zeta_{s}^{G}
$$

Comparing to the consistency condition, Eq. (3.5), implies that the mode coupling is really described by a mode coupling operator,

$$
\hat{f}_{\mathrm{NL}}=\frac{5}{6} \mathbf{x} \cdot \nabla .
$$

\footnotetext{
${ }^{5}$ We could have generated the large mode non-perturbatively with the dilation $\mathbf{x} \equiv \tilde{\mathbf{x}} e^{-\zeta_{l}}$, but since the long mode is assumed to be small, we are content to work to first order in $\zeta_{l}$.
} 
However, in a statistical sense, we can replace this operator by an equivalent numerical value. The operator $\mathbf{x} \cdot \nabla$ corresponds to a rescaling of coordinates so that, statistically, we expect it to correspond to the logarithmic derivative of the root-mean-square fluctuation in $\zeta_{s}$, i.e.

$$
f_{\mathrm{NL}}=-\frac{5}{6} \frac{d \ln \sqrt{\Delta_{\zeta}^{2}}(k)}{d \ln k}=-\frac{5}{12}\left(n_{s}-1\right) .
$$

(the minus sign follows from going to Fourier space) where $\Delta_{\zeta}^{2}(k)=\frac{k^{3} P_{\zeta}(k)}{2 \pi^{2}}$ is the dimensionless power spectrum of $\zeta$. This is how the consistency relation is commonly phrased. A more rigorous derivation of $f_{\mathrm{NL}}$ would involve explicitly calculating the effect of the mode coupling $\hat{f}_{\mathrm{NL}}$ on the squeezed limit bispectrum, or equivalently, on the modulation of the small-scale variance by the long mode, and equating this to the bispectrum predicted by the local ansatz in (3.7). Details can be found in many references, including $[1,2]$.

In the following, we will often make use of both forms for the non-Gaussianity, i.e. as an operator and as a number, but we emphasize that the latter description is only valid in a statistical sense and not at the level of Eq. (3.7). Note also that the statistical equivalence of $\mathbf{x} \cdot \nabla$ to $-d \ln \sqrt{\Delta^{2}}(k) / d \ln k$ is generally valid, but care has to be taken to use the dimensionless power spectrum $\Delta^{2}(k)$ of the field on which the operator acts. For instance, for the matter perturbations, we would get $\mathbf{x} \cdot \nabla \rightarrow-1 / 2\left(3+n_{s}\right)$. This will play an important role in the next subsection.

The key insight to be learned from this discussion is that, up to derivatives ${ }^{6}$ of $\zeta_{l}$, we can understand the effect of the long mode by performing a diffeomorphism. This insight applies in any gauge and to any quantity of interest, provided one knows how it transforms. It is this feature that is a prediction of single-field inflation which we will call the single-field consistency conditions, rather than the specific value of $f_{\mathrm{NL}}$.

The single-field consistency conditions follow from the fact that during single-field inflation there is a single "clock" (assuming the evolution has reached the inflationary attractor solution and ignoring decaying modes), see e.g. [2]. They remain valid even after inflation, as long as perturbations evolve adiabatically (so that there still is only a single clock) [29, 31]. Adiabaticity in turn is maintained as long as gravity obeys the equivalence principle and as long as scales larger than the sound horizon are considered for the long mode. Thus, since we assume general relativity, we can always apply the consistency conditions while the long mode is super-horizon, and after radiation domination we can even apply them over a large range of scales inside the horizon. We will make use of this late-universe application of the consistency conditions in the following section to derive the mode coupling of matter perturbations and to derive scale-dependent galaxy/halo bias.

The application to the late universe has recently been highlighted by [31] (based also on [30]), where they were applied directly to the matter perturbations in Newtonian gauge. The techniques used in large-scale structure follow from an observation of Weinberg [29] (which was generalized in [20]) that, in the context of single-field inflation, a long wavelength adiabatic mode can be generated by a diffeomorphism that leaves the gauge fixed. We will follow the same logic, although

\footnotetext{
${ }^{6}$ The conformal consistency conditions would allow us to extend this procedure to include one gradient of the long mode $[20,55]$. We will not include them here in the interest of pedagogy.
} 
we will specialize to synchronous gauge (see Appendix A for more details on the relation to Newtonian gauge).

\subsection{Scale-dependent bias from mode coupling}

We now turn to the calculation of scale-dependent bias in single-field inflation using the mode coupling between long and short modes. We again separate perturbations into long and short wavelength components, $\delta=\delta_{s}+\delta_{l}$, etc. We work in synchronous gauge, where the metric is given by Eq. (2.1). In this gauge, constant $\tau$ slices are hypersurfaces of constant proper time. Using the fact that galaxies live in dark matter halos, we can then express the local number density of galaxies at some time in the late universe in terms of the initial matter perturbations $\operatorname{as}^{7}$

$$
n_{g}=n_{g}\left(\delta_{l}, \sigma_{R}^{2}\right)
$$

In another gauge, different locations at constant coordinate time might be in different stages of their evolution so that the galaxy number density would also explicitly depend on the proper time fluctuations. It is thus crucial that we chose a synchronous gauge to start from (see e.g. [46]).

Eq. (3.10) says, first of all, that $n_{g}$ has an explicit dependence on the initial $\delta_{l}$. This is because the long mode affects the evolution of the small-scale perturbations and therefore the abundance of collapsed objects at a later time. Secondly, there is an explicit dependence on $\sigma_{R}^{2}$, the initial variance of small-scale fluctuations on a scale corresponding to the halos of interest. In the presence of primordial non-Gaussianity, this variance can in principle be modulated by the largescale metric perturbation $\zeta_{l}$, leading to scale-dependent bias. To quantify the scale-dependent bias in the single-field case, we thus need to compute the modulation

$$
\frac{d \sigma_{R}^{2}}{d \zeta_{l}}
$$

We choose to describe the long mode in terms of the curvature perturbation $\zeta$ because it is conserved outside of the horizon. It is common to instead write the modulation in terms of the initial Newtonian potential during matter domination, $\varphi_{l}=-3 / 5 \zeta_{l}$ (see also Appendix B). However, note that if the long-wavelength mode entered the horizon before matter-radiation equality, it is not possible to consider (super-horizon) initial conditions during matter domination.

The modulation gets two contributions:

- There is a mode coupling between $\delta_{s}$ and the long mode,

$$
\delta_{s}=\left(1+\frac{6}{5} \zeta_{l} \hat{f}_{\mathrm{NL}}^{\mathrm{eff}}\right) \delta_{s}^{G}
$$

where we have implicitly defined $\hat{f}_{\mathrm{NL}}^{\mathrm{eff}}$ analogously to the non-Gaussianity parameter for $\zeta$, cf. Eq. (3.7). This gives a modulation of the small-scale variance on a fixed coordinate scale $R$ of

$$
\left(\sigma_{R}^{2}\right)_{\varphi_{l}}=\left(1+\frac{12}{5} \zeta_{l} f_{\mathrm{NL}}^{\mathrm{eff}}\right) \sigma_{R}^{2}
$$

\footnotetext{
${ }^{7}$ We are implicitly taking an excursion set formalism approach to halo formation and we are considering galaxies living in halos of a fixed mass enclosed in an initial comoving radius $R$. However, all our conclusions are valid in general as long as galaxies live in halos and halos correspond to overdensities in the initial matter distribution.
} 
Note that $f_{\mathrm{NL}}^{\text {eff }}$ will be different than the $f_{\mathrm{NL}}$ appearing in Eq. (3.7) because the matter density has a nonlinear relation to $\zeta$. The small-scale variance is

$$
\sigma_{R}^{2}=\int d^{3} \mathbf{k}|W(k R)|^{2} P_{\delta}(k)
$$

Here, $W(k R)$ is the Fourier transform of, e.g., a spherical top-hat function enclosing a sphere with radius $R$ and $P_{\delta}(k)$ is the matter power spectrum.

- The coordinate scale on which the variance is computed should be the radius $R$ corresponding to a fixed physical lenght scale, $a(\tau) \tilde{R}$. This means that the coordinate scale $R$ is modulated by $\zeta_{l}$.

We will now show that the above two effects exactly cancel out in single-field inflation ${ }^{8}$.

In synchronous gauge, we can generate the long mode in the same way we did in comoving gauge $^{9}$ to derive the inflationary consistency conditions in Section 3.1. We again start in the local coordinate frame where the mode $\zeta_{l}$ is taken out. Here

$$
\tilde{\rho}(\tilde{\mathbf{x}}, \tau)=\bar{\rho}(\tau)\left(1+\tilde{\delta}_{s}(\tilde{\mathbf{x}}, \tau)\right)
$$

where the perturbation $\tilde{\delta}_{s}(\tilde{\mathbf{x}}, \tau)$ is independent of the long mode. Now we again apply the spatial coordinate rescaling, Eq. (3.3), that makes the long mode explicit. Since we do not transform $\tau$, and the matter density is a scalar, we have

$$
\bar{\rho}(\tau)(1+\delta(\mathbf{x}, \tau))=\rho(\mathbf{x}, \tau)=\tilde{\rho}(\tilde{\mathbf{x}}, \tau)=\bar{\rho}(\tau)(1+\tilde{\delta}(\tilde{\mathbf{x}}, \tau))
$$

so that $\delta_{s}$ transforms as a scalar $\left(\operatorname{as} \operatorname{did} \zeta_{s}\right)$ under the dilation,

$$
\delta_{s}(\mathbf{x}, \tau)=\tilde{\delta}_{s}(\tilde{\mathbf{x}}, \tau)
$$

Thus, in the coordinate system with the long mode explicit, the mode coupling takes the same form as for $\zeta$,

$$
\delta_{s}(\mathbf{x}, \tau)=\tilde{\delta}_{s}\left(\mathbf{x}\left(1+\zeta_{l}\right)\right)=\tilde{\delta}_{s}(\mathbf{x})+\zeta_{l} \mathbf{x} \cdot \nabla \tilde{\delta}_{s}(\mathbf{x})
$$

or,

$$
\delta_{s}^{(2)}=2 \zeta_{l} \mathbf{x} \cdot \nabla \tilde{\delta}_{s}
$$

Here we have expanded the matter perturbation $\delta=\delta^{(1)}+\frac{1}{2} \delta^{(2)}+\ldots$. An alternative way to derive this mode coupling, see e.g. [23-26], is to compute the second order perturbation theory solution for matter perturbations in the post-inflationary epoch and to match this solution to the consistency condition for $\zeta$ given by single-field inflation. We follow this approach for the special case of a matter dominated Universe in Appendix B and show that it yields the exact same result found in a more straightforward way above.

\footnotetext{
${ }^{8}$ In many cases, the above procedure is performed in Lagrangian coordinates. In that case, it has been shown that the consistency conditions acts trivially [41].

${ }^{9}$ The technical subtleties that have been discussed in comoving gauge and Newtonian gauge for obtaining physical solutions are not important in synchronous gauge. See Appendix A for details.
} 
Comparing this to the definition of the effective non-Gaussianity for the matter perturbation, Eq. (3.12),

$$
\delta_{s}^{(2)}=\frac{12}{5} \zeta_{l} \hat{f}_{\mathrm{NL}} \delta_{s}^{(1)}
$$

we find $\hat{f}_{\mathrm{NL}}^{\text {eff }}=\frac{5}{6} \mathbf{x} \cdot \nabla$. Statistically, the operator $\mathbf{x} \cdot \nabla$ acting on $\delta_{s}^{(1)}$ gives $-\frac{d \sqrt{\Delta_{\delta}(k)}}{d \ln k}=-\frac{1}{2}\left(3+n_{s}\right)$ (since the matter power spectrum $\Delta_{\delta} \propto k^{4} \Delta_{\zeta} \propto k^{3+n_{s}}$ ). Therefore, we get

$$
f_{\mathrm{NL}}^{\mathrm{eff}}=-\frac{5}{12}\left(3+n_{s}\right)=-\frac{5}{3}-\frac{5}{12}\left(n_{s}-1\right)=-\frac{5}{3}+f_{\mathrm{NL}} .
$$

This is the squeezed limit mode coupling result found in the literature, e.g. [22].

This leads to the following modulation of the small-scale variance on a given coordinate scale $R$,

$$
\left(\sigma_{R}^{2}\right)_{\varphi_{l}}=\left(1+\frac{12}{5} f_{\mathrm{NL}}^{\mathrm{eff}} \zeta_{l}\right) \sigma_{R}^{2}=\left(1-4 \zeta_{l}-\left(n_{s}-1\right) \zeta_{l}\right) \sigma_{R}^{2}
$$

However, the halo density should depend on the small-scale perturbation statistics on a fixed physical scale. The long-wavelength part of the spatial component of the metric is given by,

$$
\begin{aligned}
d s^{2} & =a^{2}(\tau)\left[\left(1-2 \hat{\psi}_{l}\right) d \mathbf{x}^{2}+\partial_{i} \partial_{j} \chi_{l} d x^{i} d x^{j}\right] \\
& =a^{2}(\tau)\left[\left(1+2 \zeta_{l}\right) d \mathbf{x}^{2}\right]
\end{aligned}
$$

where in the second line we have used $\hat{\psi}_{l}=-\zeta_{l}$ under the diffeomorphism defined in Eq. 3.3, and we have again neglected gradients of the long mode. Thus a physical distance is given in terms of coordinate distance in synchronous gauge by

$$
a(\tau) \tilde{R}=a(\tau)\left(1+\zeta_{l}\right) R
$$

This of course simply reflects the coordinate rescaling Eq. (3.3) we applied to generate the long mode in the first place. The small-scale variance on a fixed physical scale $a(\tau) \tilde{R}$ is now

$$
\begin{aligned}
\sigma_{\tilde{R}\left(1-\zeta_{l}\right)}^{2} & =\int d^{3} \mathbf{k}\left|W\left(k \tilde{R}\left(1+5 / 3 \varphi_{l}\right)\right)\right|^{2} P_{\delta}(k) \\
& =\int d^{3} \tilde{\mathbf{k}}|W(\tilde{k} \tilde{R})|^{2} P_{\delta}\left(\tilde{k}\left(1+\zeta_{l}\right)\right) \\
& =\sigma_{\tilde{R}}^{2}\left[1+3 \zeta_{l}+\zeta_{l} \frac{d \ln P_{\delta}(k)}{d \ln k}\right] \\
& =\sigma_{\tilde{R}}^{2}\left[1+4 \zeta_{l}+\left(n_{s}-1\right) \zeta_{l}\right] .
\end{aligned}
$$

Here, as in the above, we treat the power spectrum as a pure power law so that $d \ln P_{\delta}(k) / d \ln k=$ $n_{s}$, but is is trivial to extend this to power spectrum with running of the spectral index and higher order corrections.

The variance on the right-hand-side above, $\sigma_{\tilde{R}}^{2}$, is evaluated on a fixed coordinate scale, but still gives the small-scale variance at that scale in the synchronous gauge coordinate system. Eq. (3.22) gives the modulation of that variance by $\zeta_{l}$. Putting it all together, we find for 
the variance on a fixed, physical scale, and explicitly writing the dependence of the small-scale variance on $\zeta_{l}$,

$$
\begin{aligned}
\left(\sigma_{R=\tilde{R}\left(1-\zeta_{l}\right)}^{2}\right)_{\varphi_{l}} & =\left[1+4 \zeta_{l}+\left(n_{s}-1\right) \zeta_{l}\right]\left(\sigma_{\tilde{R}}^{2}\right)_{\varphi_{l}} \\
& =\left[1+4 \zeta_{l}+\left(n_{s}-1\right) \zeta_{l}\right]\left[1-4 \zeta_{l}-\left(n_{s}-1\right) \zeta_{l}\right] \sigma_{\tilde{R}}^{2} \\
& =\sigma_{\tilde{R}}^{2}
\end{aligned}
$$

Thus the variance has no dependence on the large-scale mode whatsoever. As a consequence, there can be no scale-dependent bias in single-field inflation ${ }^{10}$.

We clearly see that this result is trivial. The transformation to fixed physical scale simply corresponds to evaluating the small-scale variance in the frame where there is no large-scale mode, i.e. it is the variance of $\tilde{\delta}_{s}(\tilde{\mathbf{x}})$ on a scale $\tilde{R}$, which by definition does not depend on $\varphi_{l}$. Thus, we could have written down the result that $d \sigma_{R}^{2} / d \varphi_{l}=0$ from the start. Instead, we had generated the mode coupling in a less convenient coordinate system and then transformed it back to the original coordinate system.

Finally, we would like to again emphasize that these results are identical to those that we derive from perturbation theory in synchronous gauge, see Appendix B. Although the method used here (and in the next subsection) greatly simplify the calculation, all approaches give precisely the same result as long as one is careful to define the halos in terms of a fixed physical scale.

\subsection{Scale-dependent bias from direct application of consistency conditions}

A very useful observation emphasized in [31] (see also [30]) is that the procedure outlined above does not assume that the original background is homogeneous. Given a solution in a specific gauge, we can find a new solution to the equations of motion in the same gauge by performing a diffeomorphism. This procedure is linear in the long wavelength mode (and leading order in derivatives of the long mode), but is non-perturbative in the short wavelength modes. We used this feature in the previous subsections to determine the mixing between the long and short modes. However, there is no reason we cannot apply this procedure directly to the halo density field without ever discussing the biasing relative to the matter density. The halos may be determined by some non-perturbative behavior of the short modes, but to determine the influence of the long mode, all we need to know is how the halo density transforms under this diffeomorphism.

One can simply repeat the argument from the pervious section replacing $\rho$ by $\rho_{h}$, the halo number density. Working in synchronous gauge, we again find the long mode via $\mathbf{x}=\tilde{\mathbf{x}}\left(1-\zeta_{l}\right)$. The halo density transforms under a spatial diff as

$$
\bar{\rho}_{h}(\tau)\left(1+\delta_{h}(\mathbf{x}, \tau)\right)=\rho(\mathbf{x}, \tau)=\tilde{\rho}(\tilde{\mathbf{x}}, \tau)=\bar{\rho}_{h}(\tau)\left(1+\tilde{\delta}_{h}(\tilde{\mathbf{x}}, \tau)\right)
$$

so that $\delta_{s}$ transforms as a scalar $\left(\operatorname{as} \operatorname{did} \zeta_{s}\right)$ under the dilation,

$$
\delta_{h}(\mathbf{x}, \tau)=\tilde{\delta}_{h}(\tilde{\mathbf{x}}, \tau) .
$$

\footnotetext{
${ }^{10}$ The Newtonian limit of this result was also explained in [36], in which case the coordinate transformation acts trivially.
} 
Thus, in the coordinate system with the long mode explicit we find

$$
\delta_{h}(\mathbf{x}, \tau)=\tilde{\delta}_{h}\left(\mathbf{x}\left(1+\zeta_{l}\right)\right)=\tilde{\delta}_{h}(\mathbf{x})+\zeta_{l} \mathbf{x} \cdot \nabla \tilde{\delta}_{h}(\mathbf{x})=\tilde{\delta}_{h}(\mathbf{x})+\mathcal{O}\left(\delta_{h} \delta\right) .
$$

Notice that the contribution from the long mode only enters at quadratic order in the fluctuations. Therefore we can conclude at linear order that

$$
\delta_{h}^{\text {sync. }}=\tilde{\delta}_{h, S}^{\text {sync }} \rightarrow \Delta b_{\mathrm{NG}}(k)=0
$$

As discussed in Appendix A, in other gauges (e.g. Newtonian gauge) there may contributions at linear order that are proportional to $\partial_{\tau} \bar{\rho}_{h}$. However, these terms appear for densities at fixed coordinate time, rather than proper time. These linear contributions will cancel out when expressed in terms of observable quantities which are gauge invariant, as we explained in Section 2.

\section{Summary and Discussion}

Scale-dependent bias in the clustering of galaxies or halos is a powerful probe of primordial non-Gaussianity and therefore of the physics of inflation. Specifically, constraining local nonGaussianity with a precision $\sigma\left(f_{\mathrm{NL}}\right) \lesssim 1$ is, in principle, achievable with future surveys and may distinguish between single-field and multi-field inflation models. In order to correctly use such a measurement to test single-field inflation, an exact theoretical prescription for the observed galaxy overdensity, $\Delta_{g}$, is needed. This prescription must take into account the fact that $\Delta_{g}$ is measured in terms of observed redshifts and observed angular positions of galaxies, should be free of gauge ambiguities, and should include an exact prediction for the level of scale-dependent bias in single-field inflation. The latter question in particular has been a source of confusion and the main contribution of the present paper has been to clarify the calculation of this scale-dependent bias and to derive a consistent answer in several independent ways. The final result, presented in Section 3 , is that there is zero scale-dependent bias, $\Delta b_{\mathrm{NG}} \equiv 0$, if inflation is governed by a single field, leading to the complete prescription for galaxy clustering summarized in Section 2 .

Most of the confusion about scale-dependent bias in single-field models stems from the fact that matter perturbations in, for example, synchronous gauge have order-unity mode coupling, $f_{\mathrm{NL}}^{\text {eff }}=-5 / 3-5 / 12\left(n_{s}-1\right)$. Since scale-dependent bias arises from a modulation of the initial small-scale variance of matter perturbations $\left(\sigma_{R}^{2}\right)$ by a large-scale curvature perturbation $\left(\zeta_{l}\right)$, at first sight the mode coupling would thus appear to produce a scale-dependent bias corresponding to $f_{\mathrm{NL}}$ of order unity.

However, we have argued that this is not the correct interpretation. A crucial realization is that it can only be the initial variance of matter perturbations on a given physical scale, $\tilde{R}=\left(1+\zeta_{l}\right) R$, that determines the halo number density in that region at late times. While the initial variance of matter perturbations on a fixed coordinate scale does indeed see the order-unity modulation mentioned above, we have shown explicitly in Section 3.2 that when the variance is converted from being evaluated at fixed coordinate scale $R$ to being evaluated at fixed physical scale $\tilde{R}$, the terms depending on $\zeta_{l}$ cancel and the modulation is identically zero (with even the term proportional to $n_{s}-1$ vanishing).

To obtain the above result, we have derived the synchronous gauge mode coupling of matter perturbations in two ways. In Appendix B, we followed the approach common in the literature, 
which matches the second order perturbation theory solution in the post-inflationary epoch to the inflationary consistency relation. However, in Section 3.2, we derived the same result in a simpler and, we argue, more insightful way. In single-field inflation, the local effect of a large-scale curvature perturbation $\zeta_{l}$ (i.e. up to gradients) on the physics on small scales is equivalent to a simple coordinate transformation. In other words, there is no physical effect. This follows from the fact that inflation is described by a single clock in the single-field case and each region of space finds itself on the same attractor solution. It remains true after inflation as long as perturbations evolve adiabatically, which is always true on super-horizon scales (more generally, it is true on scales above the sound horizon). Using this principle, which is equivalent to the inflationary consistency condition(s), we readily generated the mode coupling of matter perturbations by a diffeomorphism. In this picture, the aforementioned cancellation of the $\zeta_{l}$ dependence of the variance became trivial: when converting to the initial variance of matter perturbations on a fixed physical scale, we were simply undoing the diffeomorphism that produced the synchronous gauge mode coupling in the first place.

Finally, in Section 3.3, we derived the absence of scale-dependent bias in an even simpler way by applying the diffeomorphism generating $\zeta_{l}$ to the halo number density directly. We demonstrated that, modulo gradients, the long mode does not affect the halo number density at first order in perturbations and thus cannot generate a scale-dependent bias.

We would like to emphasize that the absence of scale-dependent bias in single-field inflation has been to various degrees understood in the vast literature on consistency conditions in largescale structure (e.g. [31-44]). It was for example appreciated by many authors that the effect of the long mode on various quantities is zero when expressed in terms of physical scales [56], in convenient coordinates like Fermi-normal coordinates [18, 44, 57] or in Lagrangian space [41]. The main contribution of the present paper was to resolve the paradox presented by the order unity mode coupling in coordinate space that led others to conclude there must be scale-dependent bias of order unity even in the single-field context. Moreover, we provided an explicit recipe for how to use this result to compute the observed galaxy perturbations taking into account relativistic terms (see also [42]). The simple recipe we provide can be applied in any gauge and can therefore be easily incorporated into any existing calculation. We thus now have the tools to properly interpret observational constraints on scale-dependent bias even when those constraints reach a precision $\sigma\left(f_{\mathrm{NL}}\right) \lesssim 1$. If such constraints favor non-zero scale-dependent bias as carefully defined

in this paper, it would rule out the class of single-field inflation (modulo the caveats that we have assumed the inflationary evolution to have been on the attractor solution).

\section{Acknowledgements}

We thank the participants of the workshop Testing Inflation with Large Scale Structure: Connecting Hopes with Reality held at CITA, University of Toronto in October 2015 for stimulating discussions. We particularly thank Matias Zaldarriaga for several insights and for highlighting the relevance of proper physical scales in this problem. Part of the research described in this paper was carried out at the Jet Propulsion Laboratory, California Institute of Technology, under a contract with the National Aeronautics and Space Administration. This work is supported by NASA ATP grant 11-ATP-090. D.G. is supported by a NSERC Discovery Grant. 


\section{A Consistency Conditions in Newtonian and Synchronous Gauge}

In this appendix, we will review how the adiabatic modes arise from diffeomorphisms that leave Newtonian gauge fixed. We will then repeat the analysis in synchronous gauge and discuss the technical differences. Finally we will compare the description of halos in each gauge and their relation to physical observables.

To derive the consistency conditions in conformal Newtonian gauge, we will follow [31] and define the metric to take the form

$$
d s^{2}=a^{2}(\tilde{\tau})\left[-(1+2 \tilde{\Phi}) d \tau^{2}+(1-2 \tilde{\Psi}) \delta_{i j} d \tilde{x}^{i} d \tilde{x}^{j}\right]
$$

Now let us consider the transformation

$$
\begin{aligned}
\tau & =\tilde{\tau}+\epsilon(\tilde{\tau}) \\
\boldsymbol{x} & =\tilde{\boldsymbol{x}}(1-\lambda)
\end{aligned}
$$

where $\epsilon(\tau)$ is an arbitrary function of time and $\lambda$ is a constant. This transformation shifts the potentials

$$
\begin{aligned}
& \Phi(\boldsymbol{x}, \tau)=\tilde{\Phi}(\boldsymbol{x}, \tau)-\epsilon^{\prime}-\mathcal{H} \epsilon \\
& \Psi(\boldsymbol{x}, \tau)=\tilde{\Psi}(\boldsymbol{x}, \tau)-\lambda+\mathcal{H} \epsilon
\end{aligned}
$$

but leaves the gauge fixed. Under this change of coordinates, any scalar, $s$, will transform (to linear order) as

$$
s^{\mathrm{N}}(\tau, \boldsymbol{x})=\tilde{s}(\tau, \boldsymbol{x})-\epsilon(\tau) \tilde{s}^{\prime}(\tau, \boldsymbol{x}),
$$

where the ${ }^{\mathrm{N}}$ is a reminder that this is the scalar fluctuation in Newtonian gauge and ${ }^{\prime} \equiv \partial_{\tau}$. If we start from a solution to Einstein's equations, any such transformation will generate a new solution. However, most such solutions cannot be extended to physical solutions with large, but finite wavelengths $\{\epsilon(\tau), \lambda\} \rightarrow\{\epsilon(\tau, x), \lambda(x)\}$. The reason is that some of the equations of motion may simply vanish when $k=0$ and are not satisfied for any $k \neq 0$. In conformal Newtonian gauge, the only such equation is $k^{2}(\Phi-\Psi)=0$. Therefore, if we impose $\Phi=\Psi$ as an additional constraint, this transformation can be extended to a physical solution. This requires that $\epsilon^{\prime}+2 \mathcal{H} \epsilon=\lambda$ or

$$
\epsilon=\frac{\lambda}{a^{2}} \int^{\tau} d \tau^{\prime} a^{2}\left(\tau^{\prime}\right) \equiv D_{v} \lambda
$$

where $D_{v}$ is velocity growth function. In terms of $\zeta=-\Psi-H \delta \rho / \dot{\rho}$ this means that $\zeta=\tilde{\zeta}+\lambda$ (where we used $\delta \rho=-\rho^{\prime} \epsilon$ ) and therefore we see that from a differmorphism we have generated a long wavelength $\zeta$. Therefore, if we start from a FRW solution and make the identification $\lambda=\zeta_{l}$ and $\tilde{\zeta}=0$, we generate a new solution

$$
\begin{aligned}
\Phi & =-\zeta_{l}\left(D_{v}^{\prime}+\mathcal{H} D_{v}\right) \\
\Psi & =-\zeta_{l}\left(1-\mathcal{H} D_{v}\right) \\
\delta s^{\mathrm{N}} & =-\zeta_{l} D_{v} s^{\prime} .
\end{aligned}
$$


More generally, new solutions that are linear in $\zeta_{l}$ can be generated from any solution for the short modes, even non-perturbative solutions. This procedure can also be extended to include a gradient of the long mode [31] (via the conformal consistency conditions $[20,55]$ ) and to include contributions at non-linear order in the long mode $[58,59]$. We can then use this procedure to determine the mode coupling between long and short modes provided only that we neglect contributions $\mathcal{O}\left(\partial_{i} \partial_{j} \zeta_{l}\right)$.

Although the details of the above argument were specific to Newtonian gauge, such an argument will work in any gauge. For our purposes, we are are interested in synchronous gauge where the metric is taken to be

$$
d s^{2}=a^{2}(\tau)\left[-d \tau^{2}+(1-2 \hat{\psi}) \delta_{i j}^{\mathrm{K}} d x^{i} d x^{j}+\partial_{i} \partial_{j} \chi d x^{i} d x^{j}\right]
$$

It is easy to check that the transformation

$$
\boldsymbol{x}=\tilde{\boldsymbol{x}}\left(1-\zeta_{l}\right)
$$

leaves the gauge unchanged and shifts the potential

$$
\hat{\psi} \rightarrow \hat{\psi}-\zeta_{l}
$$

Unlike Newtonian gauge, there is no change to the time coordinate and therefore the transformation law for a scalar is simply

$$
\delta s^{\mathrm{S}}=0,
$$

where ${ }^{\mathrm{S}}$ is a reminder that this is the fluctuation in synchronous gauge. A priori, one may have been concerned that this was not a physical solution, as much of the gauge freedom has not been fixed by the choice of metric. Nevertheless, we can again check that in terms of $\zeta=-\hat{\psi}-H \delta \rho / \dot{\rho}$ we again have $\zeta \rightarrow \zeta+\zeta_{l}$. This shows that the usual redundancies of synchronous gauge are playing no role in this transformation as those redundancies leave $\zeta$ fixed (after all, $\zeta$ is often called "gauge invariant" because it does not transform under the redundancies of synchronous gauge). One added benefit of this gauge redundancy in synchronous gauge is that we do not need to impose an extra condition on our transformation in order to extend it to a physical solution (one can check that there are no equations of motion that vanish when $k=0$ ).

A priori, one might think that to see theses results in different gauges, we may simply look up the diffeomorphism that takes us from one gauge to another. However, since our solution is equivalent to a diffeomorphism that leaves the gauge fixed, the diffeomorphism that takes us between gauges need not preserve the form of the long wavelength mode. As a result, to understand the implications of the long mode in a given gauge, it is most useful to work directly in the appropriate gauge from the beginning and add the long mode using the relevant diffeomorphism that keeps that gauge fixed.

Now let us consider the implications for the matter density contrast $\delta$ in synchronous gauge. The total matter density $\rho(x)$ is a scalar under diffeomorphisms and therefore

$$
\rho^{\mathrm{S}}(\boldsymbol{x}, t)=\tilde{\rho}_{S}^{\mathrm{S}}(\boldsymbol{x}, t)+\zeta_{l}(\boldsymbol{x}) \boldsymbol{x} \cdot \partial \tilde{\rho}_{s}^{\mathrm{S}}+\ldots .
$$


We see that we have generated mode coupling between the short mode, $\rho_{s}$, and the long wavelength mode, $\zeta_{l}$, as described in the main text. We can perform a similar calculation in Newtonian gauge where we find

$$
\rho^{\mathrm{N}}(\boldsymbol{x}, \tau)=\tilde{\rho}_{s}^{\mathrm{N}}(\boldsymbol{x}, \tau)+\zeta_{l} D_{v} \tilde{\rho}^{\prime}+\zeta_{l} \boldsymbol{x} \cdot \partial \tilde{\rho}_{s}^{\mathrm{N}} .
$$

Defining $\delta^{\mathrm{S}, \mathrm{N}}=\frac{\rho^{\mathrm{S}, \mathrm{N}}}{\bar{\rho}}-1$ where $\bar{\rho}$ is the homogenous solution, we find

$$
\delta^{\mathrm{S}}=\delta_{s}^{\mathrm{S}}+\zeta_{L}(\boldsymbol{x}) \boldsymbol{x} \cdot \partial \delta_{s}^{\mathrm{S}} \quad \delta^{\mathrm{N}}=\delta_{S}^{\mathrm{N}}+D_{v} \zeta_{l}\left(\delta^{\prime}+\frac{\bar{\rho}^{\prime}}{\bar{\rho}}\left(1+\delta_{s}\right)\right)+\zeta_{l} \boldsymbol{x} \cdot \partial \delta_{s}^{\mathrm{N}}
$$

In both gauges, we find a coupling between the long and short modes. However, in Newtonian gauge we find additional linear terms in $\zeta_{l}$ as well as extra mode coupling contributions which arise from the time component of the diffeormorphism.

From the above procedure is should be clear that nothing required that $\rho$ was the dark matter density. It could have been any scalar quantity, including the density of halos or galaxies. Therefore, without doing any additional work, we see that at linear order in $\delta_{g}$ and $\zeta_{l}$ we have

$$
\delta_{g}^{\mathrm{S}}=\delta_{h, s}^{\mathrm{S}}+\mathcal{O}\left(\delta_{g} \zeta_{l}\right) \rightarrow \Delta b_{\mathrm{NG}}=0
$$

where $\delta_{h}^{S}$ is the physical density contrast. We emphasize that this means that, in the presence of a long wavelength mode, there is no change to density contrast of halos in synchronous gauge.

One might worry that in Newtonian gauge, we do find terms linear in $\zeta_{l}$ that would appear as scale dependent bias. However, this feature arises because we are describing the density on constant coordinate time slices, rather than in terms of some physical definition of time. If we were to compute the observed galaxy density in a redshift survey, one finds that the results appear in terms of the the gauge invariance density contrast

$$
\delta_{\text {invariant }} \equiv \delta_{g}+\frac{d \ln \bar{n}_{g}}{d \ln a} a H \delta \tau=\delta_{g}^{\mathrm{S}},
$$

where the $\delta \tau$ arises from the fluctuations in the observed redshift relative to the coordinate time and therefore the last equality follows from the feature that $\delta \tau^{\mathrm{S}} \equiv 0$. It is clear that there will be no scale dependent bias when written in terms of observable quantities in any gauge, but this is most transparent in synchronous gauge.

\section{B Second Order Perturbation Theory Calculation of Mode Coupling}

In Section 3.2, we used a diffeomorphism to directly generate the mode coupling of matter perturbations in synchronous gauge, $f_{\mathrm{NL}}^{\mathrm{eff}}=-5 / 3-5 / 12\left(n_{s}-1\right)$. This result has been derived in the literature many times by solving the second order perturbation theory equations for the matter perturbations in the post-inflationary Universe and by then matching this to the inflationary consistency condition on $\zeta$. Here, we briefly review this alternative derivation in the simple case of a matter-only Universe and show that it indeed leads to the same result, albeit through a more cumbersome calculation. We will not rederive the second order perturbation theory solution itself from scratch, but instead use well known expressions from the literature, specifically from [22, 60] (see also, e.g., [24, 61]). 
Let us expand the perturbations to second order as $\delta=\delta^{(1)}+1 / 2 \delta^{(2)}, \chi=\chi^{(1)}+1 / 2 \chi^{(2)}$, $\hat{\psi}=\hat{\psi}^{(1)}+1 / 2 \hat{\psi}^{(2)}$. In synchronous gauge, in a matter-only universe, the first order solution is then given by

$$
\begin{aligned}
\chi^{(1)} & =-\frac{\tau^{2}}{3} \varphi \\
\hat{\psi}^{(1)} & =\frac{5}{3} \varphi \\
\delta^{(1)} & =\frac{\tau^{2}}{6} \nabla^{2} \varphi
\end{aligned}
$$

(note that we have fixed the remaining gauge freedom present in synchronous gauge).

We split the solution into small- and large-scale components, $\varphi=\varphi_{s}+\varphi_{l}$. Then, to compute the mode coupling of interest, we need the second order part of the small-scale solution, but only those terms that are products of one small-scale mode and one large-scale mode, and only to zeroth order in gradients of $\varphi_{l}$. Specifically, we care about terms of order $\varphi_{l} \delta_{s}^{(1)} \sim \varphi_{l} \tau^{2} \nabla^{2} \varphi_{s}$ contributing to $\delta_{s}^{(2)}$ and terms of order $\varphi_{l} \varphi_{s}$ contributing to $\hat{\psi}^{(2)}$. The full calculation of the second order solution is cumbersome, if conceptually straightforward, and has been done for us (see, e.g., Eqs. (6) and (7) of [22]). Focusing on the relevant terms, the general result is the class of solutions ${ }^{11}$

$$
\delta^{(2)}=\frac{20}{9} \varphi_{l} \tau^{2} \nabla^{2} \varphi_{s}+\frac{1}{10} \tau^{2} \nabla^{2} \hat{\psi}^{(2)} .
$$

Note that, to the order of interest, simply solving the second order perturbation equations during matter domination allows us the freedom to choose $\hat{\psi}^{(2)}$ (at least the relevant $\mathcal{O}\left(\varphi_{l} \varphi_{s}\right)$ contribution), which is why $\delta^{(2)}$ is expressed in terms of it. For example, the equations would be solved by

$$
\begin{aligned}
\delta_{s}^{(2)} & =\frac{20}{9} \varphi_{l} \tau^{2} \nabla^{2} \varphi_{s} \\
\hat{\psi}_{s}^{(2)} & =0 .
\end{aligned}
$$

However, we can always apply a redefinition,

$$
\varphi_{s} \equiv\left(1+\varphi_{l} \hat{a}\right) \tilde{\varphi}_{s}
$$

express Eq. (B.3) in terms of $\tilde{\varphi}_{s}$ and finally drop the tilde, to get a new second order solution,

$$
\begin{aligned}
\delta_{s}^{(2)} & =\frac{20}{9} \varphi_{l} \tau^{2} \nabla^{2} \varphi_{s}+\frac{\tau^{2}}{3} \varphi_{l} \nabla^{2}\left(\hat{a} \varphi_{s}\right) \\
\hat{\psi}_{s}^{(2)} & =\frac{10}{3} \varphi_{l} \hat{a} \varphi_{s} .
\end{aligned}
$$

Thus, indeed $\delta^{(2)}=\frac{20}{9} \varphi_{l} \tau^{2} \nabla^{2} \varphi_{s}+\frac{1}{10} \tau^{2} \nabla^{2} \hat{\psi}^{(2)}$.

While the matter domination second-order perturbation theory calculation thus does not uniquely specify the solution, we can fix it by requiring that the first order solution is Gaussian, which, in terms of mode coupling, means that $\varphi_{s}^{(1)}$ is statistically independent of $\varphi_{l}^{(1)}$ (note

\footnotetext{
${ }^{11}$ Note that [60] appears to have a typo causing an inconsistency between the solution for the metric perturbation and the matter overdensity.
} 
that this is already done in the solution Eq. (6) and (7) in [22]). We do this by matching the solution to the consistency relation for $\zeta$ (see Section 3.1). Recall that the comoving curvature perturbation on constant density slices is defined as

$$
e^{2 \zeta}=(1-2 \hat{\psi})_{\delta \rho=0} .
$$

We match the perturbation theory solution Eq. (B.2) to $\zeta$, in the regime where both the large and small modes are far outside the horizon. We can then ignore gradients acting on both $\varphi_{l}$ and $\varphi_{s}$. In this limit, $\left.\hat{\psi}^{(2)}\right|_{\delta \rho=0}=\hat{\psi}^{(2)}$, the right-hand side being the solution in synchronous gauge (this follows from, e.g., Eqs. (131) and (134) of [62]). Thus,

$$
\hat{\psi}=-\zeta-\zeta^{2},
$$

or in terms of the mode coupling,

$$
\hat{\psi}_{s}=-\zeta_{s}\left(1+2 \zeta_{l}\right) .
$$

The consistency relation for $\zeta$ says

$$
\zeta_{s}=\left(1+\zeta_{l} \mathbf{x} \cdot \nabla\right) \zeta_{s}^{G} .
$$

Therefore,

$$
\hat{\psi}_{s}=-\left(1+2 \zeta_{l}+\zeta_{l} \mathbf{x} \cdot \nabla\right) \zeta_{s}^{G},
$$

where $\zeta_{s}^{G}$ is independent of $\zeta_{l}$ so that this gives the true statistical mode coupling. Identifying the first order perturbation theory solution with the Gaussian field, i.e. $\psi^{(1)}=\frac{5}{3} \varphi \equiv-\zeta^{G}$, now fixes the second order solution to

$$
\begin{aligned}
\hat{\psi}^{(2)} & =-\frac{100}{9} \varphi_{l}\left(1+\frac{1}{2} \mathbf{x} \cdot \nabla\right) \varphi_{s} \\
\delta_{s}^{(2)} & =\frac{20}{9} \varphi_{l} \tau^{2} \nabla^{2} \varphi_{s}-\frac{10}{9} \varphi_{l} \tau^{2} \nabla^{2}\left[\left(1+\frac{1}{2} \mathbf{x} \cdot \nabla\right) \varphi_{s}\right] \\
& =\frac{20}{9} \varphi_{l} \tau^{2} \nabla^{2} \varphi_{s}-\frac{10}{9} \varphi_{l} \tau^{2}\left(2+\frac{1}{2} \mathbf{x} \cdot \nabla\right) \nabla^{2} \varphi_{s} \\
& =-\frac{5}{9} \varphi_{l} \tau^{2} \mathbf{x} \cdot \nabla\left(\nabla^{2} \varphi_{s}\right) \\
& =-\frac{10}{3} \varphi_{l} \tau^{2} \mathbf{x} \cdot \nabla \delta_{s}^{(1)}=2 \zeta_{l} \mathbf{x} \cdot \nabla \delta_{s}^{(1)}
\end{aligned}
$$

with $\delta_{s}^{(1)}$ independent of the long mode by definition.

We see that this is precisely the same result for the mode coupling that we derived directly using the consistency conditions, Eq. (3.19). As explained in Section 3.2, this is equivalent to local non-Gaussianity with $f_{\mathrm{NL}}^{\mathrm{eff}}=-5 / 3-5 / 12\left(n_{s}-1\right)$. However, as detailed in Section 3.2, the mode coupling disappears when the short wavelength modes are evaluated on a fixed physical scale as opposed to a fixed (synchronous gauge) coordinate scale. 


\section{References}

[1] J. M. Maldacena, "Non-Gaussian Features of Primordial Fluctuations in Single-Field Inflationary Models," JHEP 0305 (2003) 013, arXiv:astro-ph/0210603 [astro-ph].

[2] P. Creminelli and M. Zaldarriaga, "Single field consistency relation for the 3-point function," JCAP 0410 (2004) 006, arXiv:astro-ph/0407059 [astro-ph].

[3] D. H. Lyth, C. Ungarelli, and D. Wands, "Primordial density perturbation in the curvaton scenario," Phys. Rev. D 67 no. 2, (Jan., 2003) 023503, astro-ph/0208055.

[4] M. Zaldarriaga, "Non-Gaussianities in models with a varying inflaton decay rate," Phys. Rev. D 69 no. 4, (Feb., 2004) 043508, astro-ph/0306006.

[5] M. Alvarez, T. Baldauf, J. R. Bond, N. Dalal, R. de Putter, O. Doré, D. Green, C. Hirata, Z. Huang, D. Huterer, D. Jeong, M. C. Johnson, E. Krause, M. Loverde, J. Meyers, P. D. Meerburg, L. Senatore, S. Shandera, E. Silverstein, A. Slosar, K. Smith, M. Zaldarriaga, V. Assassi, J. Braden, A. Hajian, T. Kobayashi, G. Stein, and A. van Engelen, "Testing Inflation with Large Scale Structure: Connecting Hopes with Reality," ArXiv e-prints (Dec., 2014), arXiv:1412.4671.

[6] Planck Collaboration, P. A. R. Ade, N. Aghanim, M. Arnaud, F. Arroja, M. Ashdown, J. Aumont, C. Baccigalupi, M. Ballardini, A. J. Banday, and et al., "Planck 2015 results. XVII. Constraints on primordial non-Gaussianity," ArXiv e-prints (Feb., 2015), arXiv:1502.01592.

[7] D. Baumann, M. G. Jackson, P. Adshead, A. Amblard, A. Ashoorioon, N. Bartolo, R. Bean, M. Beltrán, F. de Bernardis, S. Bird, X. Chen, D. J. H. Chung, L. Colombo, A. Cooray, P. Creminelli, S. Dodelson, J. Dunkley, C. Dvorkin, R. Easther, F. Finelli, R. Flauger, M. P. Hertzberg, K. Jones-Smith, S. Kachru, K. Kadota, J. Khoury, W. H. Kinney, E. Komatsu, L. M. Krauss, J. Lesgourgues, A. Liddle, M. Liguori, E. Lim, A. Linde, S. Matarrese, H. Mathur, L. McAllister, A. Melchiorri, A. Nicolis, L. Pagano, H. V. Peiris, M. Peloso, L. Pogosian, E. Pierpaoli, A. Riotto, U. Seljak, L. Senatore, S. Shandera, E. Silverstein, T. Smith, P. Vaudrevange, L. Verde, B. Wandelt, D. Wands, S. Watson, M. Wyman, A. Yadav, W. Valkenburg, and M. Zaldarriaga, "Probing Inflation with CMB Polarization," in American Institute of Physics Conference Series, S. Dodelson, D. Baumann, A. Cooray, J. Dunkley, A. Fraisse, M. G. Jackson, A. Kogut, L. Krauss, M. Zaldarriaga, and K. Smith, eds., vol. 1141 of American Institute of Physics Conference Series, pp. 10-120. June, 2009. arXiv:0811.3919.

[8] N. Dalal, O. Doré, D. Huterer, and A. Shirokov, "The imprints of primordial non-gaussianities on large-scale structure: scale dependent bias and abundance of virialized objects," Phys.Rev. D77 (2008) 123514, arXiv:0710.4560 [astro-ph].

[9] L. D. Ferramacho, M. G. Santos, M. J. Jarvis, and S. Camera, "Radio galaxy populations and the multitracer technique: pushing the limits on primordial non-Gaussianity," MNRAS 442 (Aug., 2014) 2511-2518, arXiv:1402.2290 [astro-ph.C0].

[10] S. Camera, M. G. Santos, and R. Maartens, "Probing primordial non-Gaussianity with SKA galaxy redshift surveys: a fully relativistic analysis," ArXiv e-prints (Sept., 2014), arXiv:1409.8286.

[11] S. Ferraro and K. M. Smith, "Using Large Scale Structure to test Multifield Inflation," ArXiv e-prints (Aug., 2014), arXiv:1408.3126.

[12] A. Raccanelli, O. Doré, and N. Dalal, "Optimization of spectroscopic surveys for testing non-Gaussianity," ArXiv e-prints (Sept., 2014), arXiv:1409.1927.

[13] D. Yamauchi, K. Takahashi, and M. Oguri, "Constraining primordial non-Gaussianity via a multitracer technique with surveys by Euclid and the Square Kilometre Array," Phys. Rev. D 90 
no. 8, (Oct., 2014) 083520, arXiv:1407.5453.

[14] R. de Putter and O. Doré, "Designing an Inflation Galaxy Survey: how to measure $\sigma\left(f_{\mathrm{NL}}\right) \sim 1$ using scale-dependent galaxy bias," ArXiv e-prints (Dec., 2014), arXiv:1412.3854.

[15] P. Creminelli, G. D'Amico, M. Musso, and J. Noreña, "The (not so) squeezed limit of the primordial 3-point function," JCAP 11 (Nov., 2011) 38, arXiv:1106.1462 [astro-ph.C0].

[16] T. Tanaka and Y. Urakawa, "Dominance of gauge artifact in the consistency relation for the primordial bispectrum," JCAP 5 (May, 2011) 14, arXiv:1103.1251 [astro-ph.C0].

[17] T. Baldauf, U. Seljak, L. Senatore, and M. Zaldarriaga, "Galaxy bias and non-linear structure formation in general relativity," JCAP 10 (Oct., 2011) 31, arXiv:1106.5507 [astro-ph.C0].

[18] E. Pajer, F. Schmidt, and M. Zaldarriaga, "The Observed Squeezed Limit of Cosmological Three-Point Functions," Phys.Rev. D88 no. 8, (2013) 083502, arXiv:1305.0824 [astro-ph.C0].

[19] B. Horn, L. Hui, and X. Xiao, "Lagrangian space consistency relation for large scale structure," ArXiv e-prints (Feb., 2015), arXiv:1502.06980 [hep-th].

[20] K. Hinterbichler, L. Hui, and J. Khoury, "Conformal Symmetries of Adiabatic Modes in Cosmology," JCAP 1208 (2012) 017, arXiv:1203.6351 [hep-th].

[21] L. Dai, E. Pajer, and F. Schmidt, "On Separate Universes," ArXiv e-prints (Apr., 2015) , arXiv:1504.00351.

[22] N. Bartolo, S. Matarrese, and A. Riotto, "Signatures of primordial non-Gaussianity in the large-scale structure of the universe," JCAP 10 (Oct., 2005) 10, astro-ph/0501614.

[23] L. Verde and S. Matarrese, "Detectability of the Effect of Inflationary Non-Gaussianity on Halo Bias," Astrophys. J Lett. 706 (Nov., 2009) L91-L95, arXiv:0909.3224.

[24] M. Bruni, J. C. Hidalgo, N. Meures, and D. Wands, "Non-Gaussian Initial Conditions in $\Lambda$ CDM: Newtonian, Relativistic, and Primordial Contributions," Astrophys. J 785 (Apr., 2014) 2, arXiv:1307.1478 [astro-ph.CO].

[25] M. Bruni, J. C. Hidalgo, and D. Wands, "Einstein's signature in cosmological large-scale structure," ArXiv e-prints (May, 2014), arXiv:1405.7006.

[26] E. Villa, L. Verde, and S. Matarrese, "General relativistic corrections and non-Gaussianity," ArXiv e-prints (Sept., 2014), arXiv:1409.4738.

[27] S. Camera, M. G. Santos, and R. Maartens, "Probing primordial non-Gaussianity with SKA galaxy redshift surveys: a fully relativistic analysis," MNRAS 448 (Apr., 2015) 1035-1043, arXiv:1409.8286.

[28] S. Camera, R. Maartens, and M. G. Santos, "Einstein's legacy in galaxy surveys," ArXiv e-prints (Dec., 2014), arXiv:1412.4781.

[29] S. Weinberg, "Adiabatic modes in cosmology," Phys.Rev. D67 (2003) 123504, arXiv:astro-ph/0302326 [astro-ph].

[30] A. L. Fitzpatrick, L. Senatore, and M. Zaldarriaga, "Contributions to the Dark Matter 3-Pt Function from the Radiation Era," JCAP 1005 (2010) 004, arXiv:0902.2814 [astro-ph.Co].

[31] P. Creminelli, J. Norea, M. Simonovic, and F. Vernizzi, "Single-Field Consistency Relations of Large Scale Structure," JCAP 1312 (2013) 025, arXiv:1309.3557 [astro-ph.C0].

[32] M. Peloso and M. Pietroni, "Galilean invariance and the consistency relation for the nonlinear squeezed bispectrum of large scale structure," JCAP 1305 (2013) 031, arXiv:1302.0223 
[astro-ph. CO].

[33] A. Kehagias and A. Riotto, "Symmetries and Consistency Relations in the Large Scale Structure of the Universe," Nucl.Phys. B873 (2013) 514-529, arXiv:1302.0130 [astro-ph.C0].

[34] M. Peloso and M. Pietroni, "Ward identities and consistency relations for the large scale structure with multiple species," JCAP 1404 (2014) 011, arXiv:1310.7915 [astro-ph.C0].

[35] P. Creminelli, J. Gleyzes, M. Simonovic, and F. Vernizzi, "Single-Field Consistency Relations of Large Scale Structure. Part II: Resummation and Redshift Space," JCAP 1402 (2014) 051, arXiv: 1311.0290 [astro-ph.CO].

[36] A. Kehagias, J. Norea, H. Perrier, and A. Riotto, "Consequences of Symmetries and Consistency Relations in the Large-Scale Structure of the Universe for Non-local bias and Modified Gravity," Nucl.Phys. B883 (2014) 83-106, arXiv:1311.0786 [astro-ph.Co].

[37] T. Nishimichi and P. Valageas, "Testing the equal-time angular-averaged consistency relation of the gravitational dynamics in N-body simulations," Phys.Rev. D90 no. 2, (2014) 023546, arXiv:1402.3293 [astro-ph.C0].

[38] B. Horn, L. Hui, and X. Xiao, "Soft-Pion Theorems for Large Scale Structure," JCAP 1409 no. 09, (2014) 044, arXiv:1406.0842 [hep-th].

[39] I. Ben-Dayan, T. Konstandin, R. A. Porto, and L. Sagunski, "On Soft Limits of Large-Scale Structure Correlation Functions," JCAP 1502 no. 02, (2015) 026, arXiv:1411.3225 [astro-ph.CO].

[40] M. Mirbabayi, M. Simonovic, and M. Zaldarriaga, "Baryon Acoustic Peak and the Squeezed Limit Bispectrum," arXiv:1412.3796 [astro-ph.CO].

[41] B. Horn, L. Hui, and X. Xiao, "Lagrangian space consistency relation for large scale structure," arXiv:1502.06980 [hep-th].

[42] A. Kehagias, A. M. Dizgah, J. Norea, H. Perrier, and A. Riotto, "A Consistency Relation for the Observed Galaxy Bispectrum and the Local non-Gaussianity from Relativistic Corrections," arXiv:1503.04467 [astro-ph.CO].

[43] T. Nishimichi and P. Valageas, "Redshift-space equal-time angular-averaged consistency relations of the gravitational dynamics," arXiv:1503.06036 [astro-ph.CO].

[44] L. Dai, E. Pajer, and F. Schmidt, "On Separate Universes," arXiv:1504.00351 [astro-ph.C0].

[45] J. Yoo, A. L. Fitzpatrick, and M. Zaldarriaga, "A New Perspective on Galaxy Clustering as a Cosmological Probe: General Relativistic Effects," Phys.Rev. D80 (2009) 083514, arXiv:0907.0707 [astro-ph.CO].

[46] D. Jeong, F. Schmidt, and C. M. Hirata, "Large-scale clustering of galaxies in general relativity," Phys.Rev. D85 (2012) 023504, arXiv:1107.5427 [astro-ph.C0].

[47] J. Yoo, N. Hamaus, U. Seljak, and M. Zaldarriaga, "Testing General Relativity on Horizon Scales and the Primordial non-Gaussianity," arXiv:1109.0998 [astro-ph.C0].

[48] J. Yoo, "General relativistic description of the observed galaxy power spectrum: Do we understand what we measure?," Phys. Rev. D 82 no. 8, (Oct., 2010) 083508, arXiv:1009.3021.

[49] A. Challinor and A. Lewis, "Linear power spectrum of observed source number counts," Phys. Rev. D 84 no. 4, (Aug., 2011) 043516, arXiv:1105.5292 [astro-ph.Co].

[50] M. Bruni, R. Crittenden, K. Koyama, R. Maartens, C. Pitrou, and D. Wands, "Disentangling 
non-Gaussianity, bias, and general relativistic effects in the galaxy distribution," Phys. Rev. D 85 no. 4, (Feb., 2012) 041301, arXiv:1106.3999 [astro-ph.Co].

[51] C. Bonvin and R. Durrer, "What galaxy surveys really measure," Phys. Rev. D 84 no. 6, (Sept., 2011) 063505, arXiv:1105.5280 [astro-ph.C0].

[52] D. Bertacca, R. Maartens, A. Raccanelli, and C. Clarkson, "Beyond the plane-parallel and Newtonian approach: wide-angle redshift distortions and convergence in general relativity," JCAP 10 (Oct., 2012) 25, arXiv:1205.5221 [astro-ph.Co].

[53] J. M. Bardeen, P. J. Steinhardt, and M. S. Turner, "Spontaneous creation of almost scale-free density perturbations in an inflationary universe," Phys. Rev. D 28 (Aug., 1983) 679-693.

[54] D. S. Salopek and J. R. Bond, "Nonlinear evolution of long-wavelength metric fluctuations in inflationary models," Phys. Rev. D 42 (Dec., 1990) 3936-3962.

[55] P. Creminelli, J. Norena, and M. Simonovic, "Conformal consistency relations for single-field inflation," JCAP 1207 (2012) 052, arXiv:1203.4595 [hep-th].

[56] L. Senatore and M. Zaldarriaga, "A Note on the Consistency Condition of Primordial Fluctuations," JCAP 1208 (2012) 001, arXiv:1203.6884 [astro-ph.C0].

[57] T. Baldauf, U. Seljak, L. Senatore, and M. Zaldarriaga, "Galaxy Bias and non-Linear Structure Formation in General Relativity," JCAP 1110 (2011) 031, arXiv:1106.5507 [astro-ph.CO].

[58] M. Mirbabayi and M. Zaldarriaga, "Double Soft Limits of Cosmological Correlations," JCAP 1503 no. 03, (2015) 025, arXiv:1409.6317 [hep-th].

[59] A. Joyce, J. Khoury, and M. Simonovic, "Multiple Soft Limits of Cosmological Correlation Functions," JCAP 1501 no. 01, (2015) 012, arXiv:1409.6318 [hep-th].

[60] S. Matarrese, S. Mollerach, and M. Bruni, "Relativistic second-order perturbations of the Einstein-de Sitter universe," Phys. Rev. D 58 no. 4, (Aug., 1998) 043504, astro-ph/9707278.

[61] N. Bartolo, S. Matarrese, O. Pantano, and A. Riotto, "Second-order matter perturbations in a $\Lambda \mathrm{CDM}$ cosmology and non-Gaussianity," Classical and Quantum Gravity 27 no. 12, (June, 2010) 124009, arXiv:1002.3759.

[62] N. Bartolo, E. Komatsu, S. Matarrese, and A. Riotto, "Non-Gaussianity from inflation: theory and observations," Physics Reports 402 (Nov., 2004) 103-266, astro-ph/0406398. 